'Departamento de Imagenología, Facultad de Medicina Clínica Alemana-Universidad del Desarrollo. Santiago, Chile. aResidente de Radiología.

Trabajo no recibió financiamiento Los autores declaran no tener conflictos de interés.

Recibido el 17 de febrero de 2020, aceptado el 27 de agosto de 2020.

Correspondencia a: Dra Daniela Barahona Zuleta Av. Vitacura 5951, Vitacura. Santiago, Chile. danibarahona@gmail.com

\section{Actualización en el uso del PET/CT en sarcoma de Ewing, a propósito de un caso}

\author{
DANIELA BARAHONA Z. ${ }^{1}$, ISABEL ADLERSTEIN L. ${ }^{1, a}$, \\ MARÍA IGNACIA FAURE R.,a
}

\section{Use of PET/CT for staging of Ewing's sarcoma. Report of one case}

The use of positron emission tomography with computed tomography (PET/ $C T)$ for adult cancer patients is widespread, however, its use in pediatric patients is limited by fear of radiation, monetary cost and lack of awareness of its greater sensitivity in the evaluation of some types of tumors. Ewing's sarcoma is one of the primary pediatric malignancies in which PET/CT with ${ }^{18} \mathrm{~F}$-labeled fluorodeoxyglucose $\left({ }^{18} F-F D G\right)$ has demonstrated greater sensitivity in the evaluation of bone metastases compared to scintigraphy, as well as in the evaluation of treatment response. We report a 13 years old female consulting for retrosternal pain. A chest CT scan showed an infiltrating mass originating in the sternum. A biopsy confirmed the presence of an Ewing sarcoma. The tumor was staged with PET/CT which showed multiple bone lesions not visible in previous studies.

(Rev Med Chile 2020; 148: 1504-1507)

Key words: Bone Marrow; Fluorodeoxyglucose F18; Positron Emission Tomography Computed Tomography; Radionuclide Imaging; Sarcoma, Ewing.
$\mathrm{E}$ 1 sarcoma de Ewing es el segundo tumor óseo más frecuente en la edad pediátrica, dando cuenta aproximadamente de $3 \%$ de todos los cánceres en este grupo etario. Aproximadamente $25 \%$ de los pacientes presenta diseminación a distancia al momento del diagnóstico, la cual suele ser hematógena con compromiso típicamente pulmonar y óseo, menos frecuentemente, cardiaco o cerebral ${ }^{1}$.

La etapificación clásica incluye tomografía computada de tórax para la detección de nódulos pulmonares, cintigrafía ósea y biopsia de médula ósea, siendo el tumor primario evaluado idealmente con resonancia magnética; sin embargo, múltiples estudios han demostrado la mayor sensibilidad del PET/CT FDG-F18 en la detección de metástasis óseas en comparación con la cintigrafía e incluso algunos estudios plantean la posibilidad de omitir la biopsia de médula ósea en presencia de un PET sin evidencia de diseminación ${ }^{2}$.
Mediante un caso clínico se pretende ejemplificar la utilidad del PET/CT FDG-F18 en la etapificación y control de tratamiento en una paciente con sarcoma de Ewing.

\section{Caso clínico}

Paciente de género femenino de 13 años que consulta en servicio de urgencia por dolor retroesternal opresivo de 24 hrs de evolución, EVA 6/10 y signos vitales normales. Al examen físico se palpó aumento de volumen paraesternal derecho que impresionó de origen óseo-cartilaginoso, sospechándose una costocondritis. Se solicitó una radiografía de esternón, la cual no evidenció alteraciones, indicándose manejo médico. La paciente vuelve a consultar un mes y medio después con mayor aumento de volumen, solicitándose una tomografía computada de tórax 

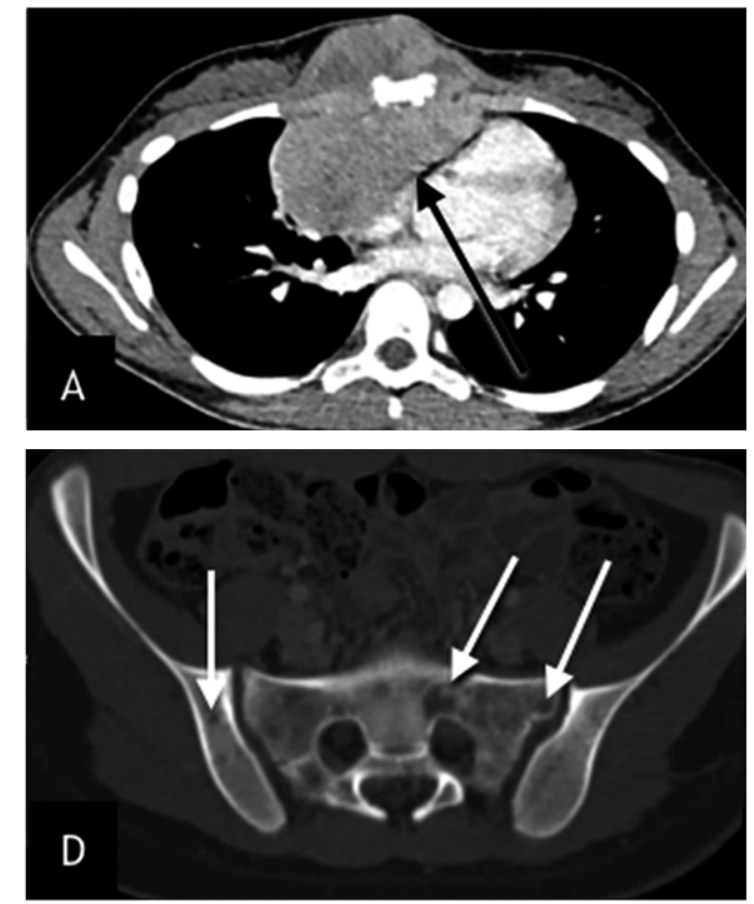
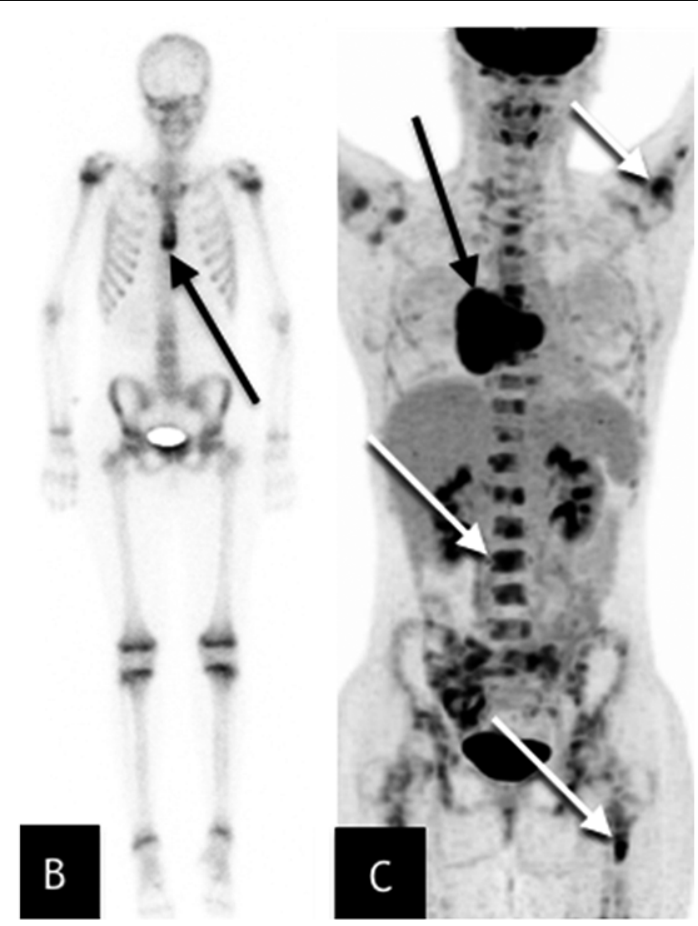

Figura 1. A: Tomografía computada de tórax contrastada axial donde se observa una masa de partes blandas (flecha negra) que rodea e infiltra el esternón y desplaza al corazón. B: imagen MiP (maximum intensity projection) de PET/CT FDG-F18 que demuestra el tumor primario hipermetabólico (flecha negra) y múltiples focos hipermetabólicos óseos (flechas blancas). C: Cintigrama óseo con Tecnecio 99m-MDP en el cual se demuestra captación focal en el esternón (flecha negra) debido a actividad osteoblástica en el sitio del tumor primario, sin otros focos captantes patológicos en el resto del cuerpo. D: Tomografía computada axial de pelvis donde se reconocen múltiples lesiones osteolíticas que se correlacionan con los focos hipermetabólicos visibles en el PET (flechas blancas).

para su evaluación, la cual detectó una masa de partes blandas infiltrativa, originada del esternón (Figura 1, a), altamente sospechosa de sarcoma óseo, sin evidencia de metástasis pulmonares. Se realizó biopsia del tumor primario, confirmando un sarcoma de Ewing de origen esternal. Como estudio de etapificación inicial se indicó cintigrafía ósea, la cual solo detectó captación esternal focal debido a actividad osteoblástica en el sitio del tumor primario (Figura 1, b) y una biopsia de cresta iliaca unilateral con resultado negativo. Con posterioridad se solicitó PET/CT FDG-F18, previo al inicio de la terapia, el cual además del tumor primario esternal hipermetabólico, detectó múltiples pequeñas lesiones osteolíticas hipercaptantes y focos hipermetabólicos en la médula ósea compatible con localizaciones secundarias (Figura $1 \mathrm{c}, \mathrm{d}$ ).
Luego de cuatro meses de inicio de la quimioterapia se reevaluó con PET/CT, el cual demostró respuesta metabólica completa tanto de la lesión tumoral primaria como de las lesiones óseas e infiltración medular (Figura 2).

La madre de la paciente otorgó el consentimiento para la publicación del caso clínico.

\section{Discusión}

El sarcoma de Ewing corresponde a una neoplasia pediátrica agresiva, parte de la familia de tumores del tipo células pequeñas redondas azules. Dentro de los principales factores de sobrevida se encuentra la existencia de metástasis, presente en $25 \%$ de los pacientes al momento del diagnóstico, siendo de suma relevancia su correcta detección ${ }^{3}$. La diseminación hematógena es la más habitual, 


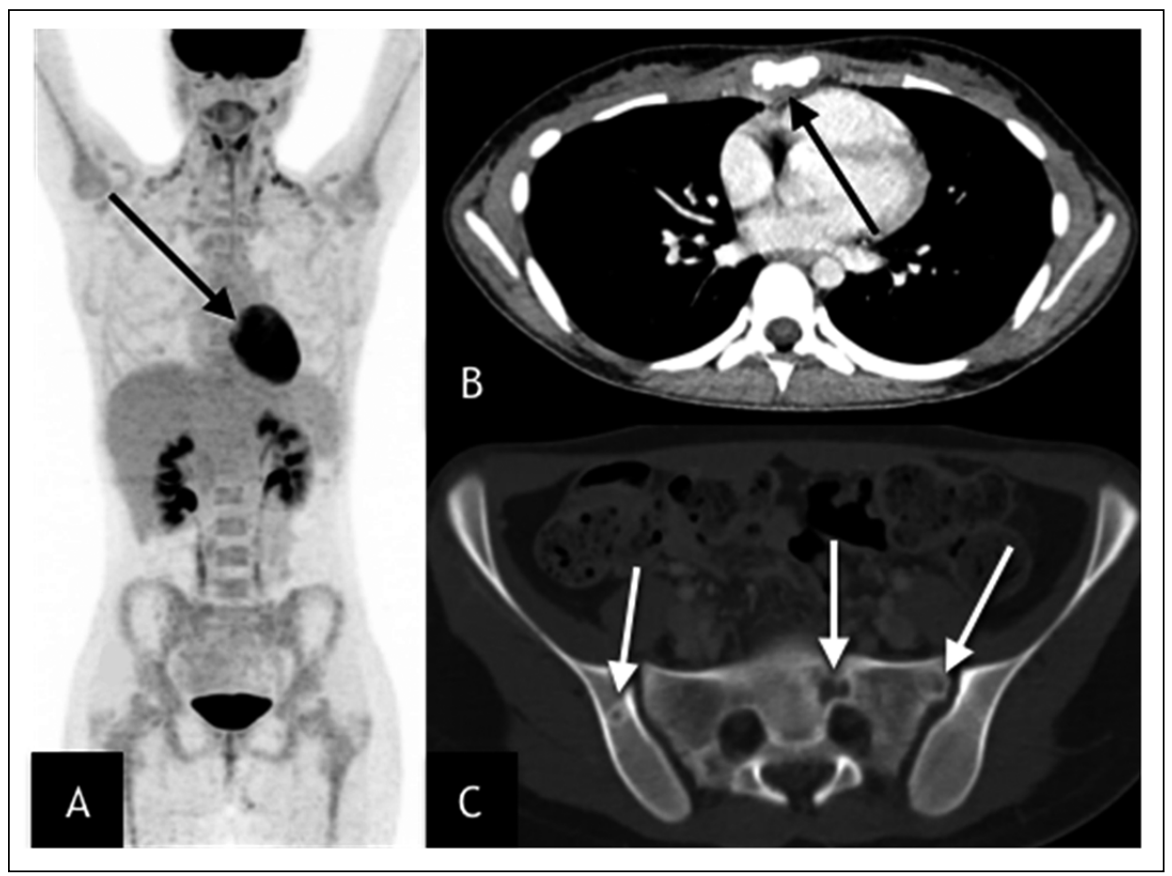

Figura 2. A: Imagen MIP de PET/CT FDG-F18 donde ya no son visibles los focos hipermetabólicos óseos ni el tumor primario, solo captación miocárdica fisiológica (flecha negra). B: Tomografía computada axial de tórax con contraste donde se observa solo escaso tejido de partes blandas retroesternal residual (flecha negra). C: Tomografía computada axial de pelvis en ventana ósea donde se observa aparición de un halo de esclerosis en las lesiones líticas (flechas blancas) como fenómeno reparativo, ninguna de ellas hipermetabólica en el PET.

siendo, en orden de frecuencia, las metástasis pulmonares, óseas y en médula ósea las más típicas, detectadas mediante tomografía computada de tórax, cintigrafía ósea y biopsia de médula ósea respectivamente ${ }^{4}$. Sin embargo, en la actualidad, múltiples estudios han demostrado la mayor sensibilidad de técnicas híbridas como el PET/CT en la detección de metástasis en hueso y médula ósea, pero también en tejidos blandos, ya que corresponde a un tipo de tumor con alto consumo glucídico y, por lo tanto, muy hipermetabólico ${ }^{5}$.

En la detección de metástasis pulmonares se ha descrito menor sensibilidad del PET/CT en comparación con la tomografía computada ${ }^{6}$, sin embargo, ambos estudios son equivalentes si se incorpora una tomografía en inspiración durante la adquisición del PET/CT.

En nuestro caso el estudio inicial de la paciente se realizó con una cintigrafía ósea, la cual solo logró detectar las alteraciones locales en relación con el tumor primario, pero no así la extensa diseminación en el resto del esqueleto. Esto se explica porque la cintigrafía detecta actividad osteoblástica, no las células tumorales propiamente tales, su sensibilidad disminuye en lesiones líticas sin actividad reparativa inicial y no es capaz de detectar la infiltración medular; su mayor rendimiento es en la evaluación de lesiones escleróticas.

La biopsia de médula ósea tampoco logró identificar infiltración medular, la cual sí fue demostrada en el PET/CT; si la infiltración medular es parcheada, como en nuestro caso, sin afectación de las crestas iliacas, sitio habitual de la toma de muestra, el rendimiento de la biopsia será menor. El PET/CT es un buen método para guiar la biopsia de médula ósea a un sitio hipermetabólico, el cual puede encontrarse alejado de las crestas iliacas, y así aumentar su rendimiento en la detección de infiltración medular ${ }^{7}$.

Se ha estudiado el uso del PET/CT en la evaluación de la respuesta a tratamiento en el sarcoma de Ewing, siendo reconocida su utilidad como factor pronóstico y su buena correlación con la respuesta tumoral ${ }^{8}$. 
La National Comprehensive Cancer Network (NCCN) recomienda en su guía del año 2020 el uso del PET-CT en el manejo inicial y en la evaluación de la respuesta a tratamiento del sarcoma de Ewing.

\section{Conclusión}

En la actualidad las guías internacionales recomiendan el uso del PET/CT FDG-F18 en la etapificación y evaluación de respuesta a tratamiento del sarcoma de Ewing debido a su mayor sensibilidad y precisión en comparación con otros métodos de imágenes.

\section{Referencias}

1. Bernstein M, Kovar H, Paulussen M, Randall RL, Schuck A, Teot LA, et al. Ewing's sarcoma family of tumors: current management. Oncologist 2006; 11 (5): 503-19.

2. Inagaki C, Shimoi $\mathrm{T}$, Sumiyoshi Okuma H, Kawachi A, Sudo K, Shimomura A, et al. Bone marrow examination in patients with Ewing sarcoma/peripheral primitive neuroectodermal tumor without metastasis based on 18F-fluorodeoxyglucose positron emission tomography/computed tomography. Med Oncol 2019; 36 (7): 58.

3. Ladenstein R, Pötschger U, Le Deley MC, Whelan J, Paulussen $\mathrm{M}$, Oberlin $\mathrm{O}$, et al. Primary disseminated multifocal Ewing sarcoma: results of the Euro-EWING 99 trial. J Clin Oncol 2010; 28 (20): 3284-91.

4. Balamuth NJ, Womer RB. Ewing's sarcoma. Lancet Oncol 2010; 11 (2): 184-92.

5. Newman EN, Jones RL, Hawkins DS. An evaluation of [F-18]-fluorodeoxy-D-glucose positron emission tomography, bone scan, and bone marrow aspiration/biopsy as staging investigations in Ewing sarcoma. Pediatr Blood Cancer 2013; 60 (7): 1113-7.

6. Quartuccio N, Fox J, Kuk D, Wexler LH, Baldari S, Cistaro A, et al. Pediatric Bone Sarcoma: Diagnostic Performance of 18F-FDG PET/CT Versus Conventional Imaging for Initial Staging and Follow-Up. AJR Am J Roentgenol 2014; 204 (1): 153-60.

7. Kasalak Ö, Glaudemans AWJM, Overbosch J, Jutte PC, Kwee TC. Can FDG-PET/CT replace blind bone marrow biopsy of the posterior iliac crest in Ewing sarcoma? Skeletal Radiol 2018; 47 (3): 363-7.

8. Hawkins DS, Schuetze SM, Butrynski JE, Rajendran JG, Vernon CB, Conrad EU, et al. [18F]Fluorodeoxyglucose positron emission tomography predicts outcome for Ewing sarcoma family of tumors. J Clin Oncol 2005; 23 (34): 8828-34. 